\begin{tabular}{|c|c|c|}
\hline & $\begin{array}{l}\text { http://dergipark.org.tr/tr/pub/anatolianbryology } \\
\text { DOI: 10.26672/anatolianbryology.657601 }\end{array}$ & $\begin{array}{c}\text { Anatolian Bryology } \\
\text { Anadolu Briyoloji Dergisi } \\
\text { Research Article } \\
\text { e-ISSN:2458-8474 Online }\end{array}$ \\
\hline \multicolumn{3}{|c|}{ 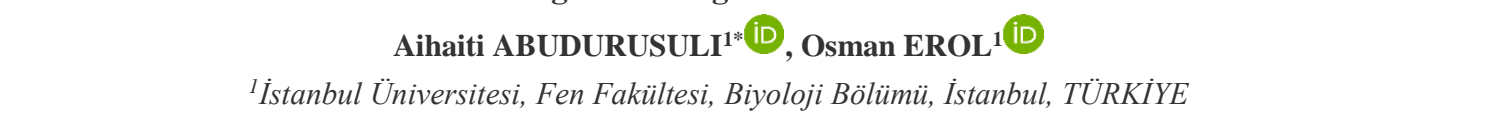 } \\
\hline Received: 12.10.2019 & Revised: 27.12 .2019 & epted: 10.03.2020 \\
\hline
\end{tabular}

Öz

Bu çalışmada, Batı Tanrı Dağlarındaki Marchantiophyta üyeleri araştırılmıştır. Araştırma alanından (Sincan Uygur Özerk Bölgesi, Çin) 2012-2015 yılları arasında toplanan 323 adet örnek teşhis edilerek Marchantiophyta bölümünden 17 familyaya ait 21 cins ve bu cinslere ait 41 tür tespit edilmiştir. Teşhis edilen türlerin substrat tipleri ve bu substrat tiplerine ait olan örnek sayıları belirlenmiştir. Her bir substrat tipine ait olan örnek sayılarının toplam örnek sayısına oranları hesaplanmıştır. Buna göre toplanan 323 örnekten 142'si “ölü ağaç kütükleri” üzerinden toplanmıştır. Bu örnekler toplanan tüm örneklerin \%43,61'ini oluşturmaktadır. Diğer örnekler sırayla 95 örnek $(\% 29,60)$ "kaya üzerinden”, 51 örnek (\%15,89) “toprak üzerinden”, 23 örnek $(\% 7,16)$ “ağaç üzerinden”, 11 örnek $(\% 3,43)$ "Islak Toprak Üzerinden” ve 1 örnek $(\% 0,31)$ "islak kaya üzerinden” toplanmıştır. İçerdikleri tür sayısı yönünden incelendiğinde bu bölgede en fazla taksonun 8 tür ile Lophoziaceae familyasında olduğu görülmektedir. Bu familyaya ait türlerin tüm türlere oranı \%19,51'dir. Bölgedeki en fazla türe sahip diğer iki familya dörder türle Aneuraceae ve Scapaniaceae familyalarıdır ve bunların diğer türlere oranı \%9,76'dır.

Anahtar kelimeler: Flora, Marchantiophyta, Lophoziaceae, Aneuraceae, Scapaniaceae.

\title{
Contribution to the Liverwort Flora of Western Tian Shan Mountains
}

\section{Abstract}

In this study, floristical investigations on members of Marchantiophyta in West Tian Shan Mountains were carried out. In this study, 323 liverwort specimens were collected in West Tian Shan Mountains of Xin Jiang, China, between 20122015. After identification of specimens' 41 liverwort (Marchantiophyta) taxa belonging 17 families and 21 genera are reported. The habitat types of the species identified and the specimen numbers belonging to these habitat types were determined. The ratios of the specimen numbers of each habitat type to the total number of specimens were calculated. According to this, 142 of 323 samples were collected from "dead tree logs". These samples comprise $43.61 \%$ of all the samples collected. Of the other specimens 95 were collected (29.60\%) from "rocks", 51 (15.89\%) from "soil", 23 (7.16\%) from "trees", 11 (3.43\%) from "wet soil" and $1(0.31 \%)$ from "wet rock". In terms of species number, Lophoziaceae, with 8 species is the richest family in this area. The species ratio of the Lophoziaceae family and others is $19.51 \%$. The other two families with the highest number of species are Aneuraceae and Scapaniaceae with 4 species, and the rate of these families to other families at the study area is $9.76 \%$.

Keywords: Flora, Marchantiophyta, Lophoziaceae, Aneuraceae, Scapaniaceae.

*Corresponding author: abdullaahat@gmail.com
C 2020 All rights reserved / Tüm haklart saklıdır.

To cite this article: Abudurussuli A. Erol O. 2020. Contribution to the Liverwort Flora of Western Tian Shan Mountains. Anatolian Bryology. 6:1, 8-18.

(c) () () () This work is licensed under a Creative Commons Attribution-Non Commercial 4.0 International License. 


\section{Giriș}

Çin'in kuzeybatısında yer alan ve eyalet bölgesi olan Sincan Uygur Özerk Bölgesi dünyanın biyoçeşitlilik açısından en zengin bölgelerinden biridir. Bu bölge genelde dağ, yayla ve havzalardan oluşmaktadır. Bölgenin biyoçeşitlilik açısından zenginliklerinin gerektiği şekilde değerlendirilip sağlıklı ve verimli bir şekilde aktarılabilmesi önem taşımaktadır. Biyolojik zenginliklerin gerçek potansiyelinin ortaya çıkartılması ve korunmasına yönelik yapılan taksonomik ve ekolojik çalışmalar bu amaca ulaşmada çok önemli bir ayağı oluşturmaktadır (Gupur ve ark., 2014).

Sincan Uygur Özerk Bölgesinde yapılan floristik ve taksonomik çalışmalara bakıldığında, büyük oranda tohumlu bitkiler üzerine yoğunlaşıldığ1 görülmektedir. "Flora Xinjiangensis" (Yang Chang You, 1993) adlı eserde Sincan Eğrelti ve Tohumlu Bitkiler Floraları 6 cilt halinde yazılmış olmasına rağmen Sincan briyofitleri üzerine yapılan floristik ve ekolojik çalışmalar yetersizdir ve son yıllarda bu konuda çalışmalar yapılmaya başlanmıştır. Karayosunları, ciğerotları, mantarlar ve likenlerle ilgili bölgesel ve yerel çalışmalar devam etmektedir. Ancak henüz Sincan'daki gerçek potansiyeli yansitacak olan flora çalışmaları yapılmamıştır.

Araştırma alanı olarak seçilen Batı Tanrı Dağı bölgesi, Sincan Tanrı Dağı'nın batısında yer almaktadır. Tanrı Dağları, batıda Özbekistan ile başlayıp Kazakistan ve Kırgızistan'dan geçerek doğuda Çin'in Sincan Uygur Özerk Bölgesi’nin Kumul şehrine kadar giden, Orta Asya'nın en büyük dağ topluluklarından biridir. Tanrı Dağları'nın toplam uzunluğu 2500 km'dir ve bunun 1700 km'si Sincan'dadır. En yüksek noktası olan Tömür Zirvesi (Sincan) 7435.3 metreye ulaşır (Hu Ru Ji ve ark., 2004). Bu çalışma kapsamında Tanrı Dağları'nın Sincan'da yer alan bölümü çalışılmıştır.

Çalışma alanı olarak seçilen Sincan Batı Tanrı Dağları' ndaki (Dokuztara, Moğulküre, Tekes, Nilka, Gulca, Korgas, Künes, Çapçal ve Hoçing ilçeleri) ciğerotları hakkında bugüne kadar detaylı bir çalışma yapılmamıştır. Bu çalışmanın amacı, Sincan Batı Tanrı Dağı'ndaki ciğerotlarının tür çeşitliliğini belirlemek, taksonların dağılım durumunu ortaya koymak ve Sincan Batı Tanrı dağındaki ciğerotları florasını ortaya çıkarmaktır. Bu çalışma, Sincan Uygur Özerk Bölgesi'ndeki gerçek ciğerotu potansiyelinin ortaya çıkarılmasına ve ileride yazılacak olan Sincan Uygur Özerk Bölgesi Ciğerotları Florası'na katkıda bulunacaktır.

\section{2. Çalışma Alanı Coğrafik Konumu}

Araştırma alanı olarak seçilen Batı Tanrı Dağı bölgesi, Sincan Tanrı Dağı'nın batısında yer almaktadır. Araştırma alanının büyük bir kısmı İli Kazak Özerk ili ve az bir kısmı ise Bayanglin Moğul Özerk ilinde bulunan Hoçing ilçesi sınırları içerisindedir (Şekil 1). 


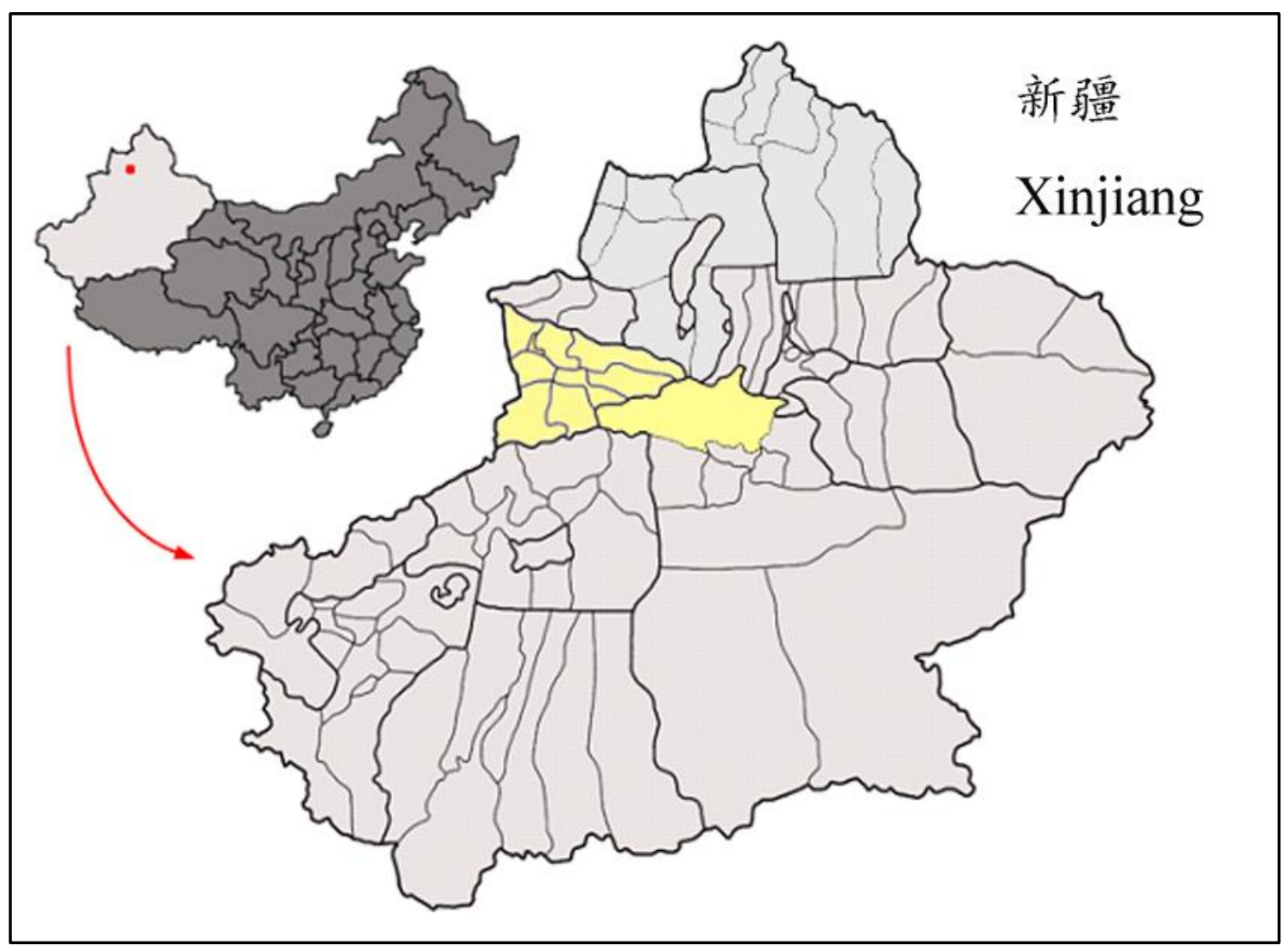

Şekil 1. Araştırma alanının konumu (sarıya boyanmış bölge) (URL1).

\subsection{Vejetasyonu}

Batı Tanrı Dağı vejetasyon tiplerine bakıldığında, aşağıdaki vejatasyon tipleri görülmektedir:

a. Dağlık çöl bölgesi: Rakım 700-1000 m, bu bölgede çoğunlukla Artemisia spp. Ceratocarpus arenarius L., Salsola spp. taksonlarına rastlanır (Zhang, 2002).

b. Dağlık çayır bölgesi: Rakım 1000-1500 m, bu bölgede ise Bromus japonicus Thunb. ex Murr., $B$. sylvaticum (Huds) Beauv., Stipa capillata, Festuca rupicola Heuff. ve Spiraea hypericifolia L. türlerine rastlanır (Zhang, 2002).

c. Orman bölgesi: Rakım 1500-2200 m aras1, bu bölgede ağırlıklı olarak Salix xerophila Flod., Picea schrenkina Fisch. \& C.A.Mey., Betula tianschanica Rupr., Populus L. türlerine rastlanır(Zhang, 2002). d. Subalpin step bölgesi: Rakım 2200-2900 m arası, bu bölgede çoğunlukla Alchemilla cyrtopleura Juz., Phlomoides oreophila (Kar. \& Kir.) Adylov, Kamelin \& Makhm., ve Carex spp. taksonlarına rastlanır (Zhang, 2002).

e. Alpin çayır bölgesi: Rakım 2900 m den yüksek alanlar, bu bölgede sıklıkla Artemisia Linn. cinsine ait türlere, Kobresia capillifolia (Decaisne) C.B.Clarke, ve Carex atrata L., C. melanantha C.A.Mey. türlerine rastlanır (Zhang, 2002).

\section{Materyal ve Metot}

Araştırma materyalini oluşturan ciğerotu örnekleri 2012- 2015 yılları arasında farklı tarihlerde ve vejetasyonun farklı dönemlerinde Batı Tanrı Dağları'ndan belirlenen 21 istasyondan toplanmıştır (Tablo 1). 
Tablo 1: Örneklerin toplandığı istasyonlar ve özellikleri.

\begin{tabular}{|c|c|c|c|c|}
\hline İst. No. & Tarih & Enlem-Boylam & Rakım (m) & Lokalite \\
\hline 1 & $\begin{array}{l}25.06 .2012 \\
29.06 .2012\end{array}$ & $\begin{array}{l}\mathrm{N} 44^{\circ} 08^{\prime} 11.2^{\prime \prime} \\
\mathrm{E} 81^{\circ} 42^{\prime} 43.1^{\prime \prime}\end{array}$ & $1400-1610$ & $\begin{array}{l}\text { Gulca ilçesi A wu zan aktaş turist } \\
\text { bölgesi }\end{array}$ \\
\hline 2 & $\begin{array}{l}10.06 .2014 \\
12.06 .2014\end{array}$ & $\begin{array}{l}\mathrm{N} 43^{\circ} 25^{\prime} 28.89^{\prime \prime} \\
\mathrm{E} 81^{\circ} 01^{\prime} 24.04^{\prime \prime}\end{array}$ & $1770-2280$ & Çapçal ilçesi çion bu la \\
\hline 3 & $\begin{array}{l}15.06 .2014 \\
09.06 .2015\end{array}$ & $\begin{array}{l}\mathrm{N} 42^{\circ} 33^{\prime} 83.4^{\prime \prime} \\
\mathrm{E} 80^{\circ} 19^{\prime} 89.7^{\prime \prime}\end{array}$ & $2160-2260$ & Moğolküre ilçesi Muzat nehiri kenarı \\
\hline 4 & 18.06 .2014 & $\begin{array}{l}\mathrm{N}_{4} 2^{\circ} 32^{\prime} 07.44^{\prime \prime} \\
\mathrm{E} 81^{\circ} 11^{\prime} 01.26^{\prime \prime}\end{array}$ & $2280-2640$ & Moğolküre Akyaz ka bu ting su \\
\hline 5 & $\begin{array}{l}20.06 .2014 \\
21.06 .2014 \\
\end{array}$ & $\begin{array}{l}\mathrm{N} 42^{\circ} 54 ' 53.11^{\prime \prime} \\
\mathrm{E} 82^{\circ} 11^{\prime} 58.59^{\prime \prime}\end{array}$ & $2010-2030$ & Tekes ilçesi çion ku şı tai \\
\hline 6 & 22.06 .2014 & $\begin{array}{l}\text { N42 } 54 ' 27.54^{\prime \prime} \\
\text { E81 } 59 \text { '28.42" }\end{array}$ & 1985 & Tekes ilçesi şiao ku şı tai \\
\hline 7 & $\begin{array}{l}23.06 .2014 \\
24.06 .2014\end{array}$ & $\begin{array}{l}\text { N42 } 47^{\circ} 13.32^{\prime \prime} \\
\text { E81 } 54^{\circ} 12.45^{\prime \prime}\end{array}$ & $1600-1740$ & Tekes ilçesi ku ke su wen quan \\
\hline 8 & $\begin{array}{l}26.06 .2014 \\
27.06 .2014 \\
\end{array}$ & $\begin{array}{l}\mathrm{N} 43^{\circ} 02^{\prime} 31.83^{\prime \prime} \\
\mathrm{E} 82^{\circ} 33^{\prime} 22.33^{\prime \prime}\end{array}$ & $1890-2100$ & Dokuztara ilçesi tarim \\
\hline 9 & $\begin{array}{l}28.06 .2014 \\
29.06 .2014\end{array}$ & $\begin{array}{l}\mathrm{N} 43^{\circ} 17^{\prime} 36.51^{\prime \prime} \\
\mathrm{E} 83^{\circ} 04^{\prime} 36.13^{\prime \prime}\end{array}$ & $1500-1520$ & Dokuztara ilçesi bo tu \\
\hline 10 & 02.07 .2014 & $\begin{array}{l}\mathrm{N}^{\prime} 3^{\circ} 22^{\prime} 10.31^{\prime \prime} \\
\mathrm{E} 84^{\circ} 21^{\prime} 33.15^{\prime \prime}\end{array}$ & 1760 & Künes ilçesi künes orman alanı \\
\hline 11 & 03.07 .2014 & $\begin{array}{l}\mathrm{N}_{4} 3^{\circ} 13^{\prime} 55.65^{\prime \prime} \\
\mathrm{E} 84^{\circ} 38^{\prime} 23.38^{\prime \prime}\end{array}$ & 2175 & Hoçing ilçesi gong nai si nan gou \\
\hline 12 & 04.07 .2014 & $\begin{array}{l}\mathrm{N}_{4} 3^{\circ} 15^{\prime} 55.45^{\prime \prime} \\
\mathrm{E} 84^{\circ} 18^{\prime} 18.52^{\prime \prime}\end{array}$ & 1673 & Hoçing ilçesi gong nai si 309 \\
\hline 13 & 06.07 .2014 & $\begin{array}{l}\mathrm{N}^{\prime} 3^{\circ} 36^{\prime} 04.26^{\prime \prime} \\
\mathrm{E} 83^{\circ} 52^{\prime} 02.32^{\prime \prime}\end{array}$ & $1760-2240-2745$ & Nilka ilçesi yi şıng bu gu \\
\hline 14 & 07.07 .2014 & $\begin{array}{l}\mathrm{N}_{4}^{\circ} 04^{\circ} 22.13^{\prime \prime} \\
\mathrm{E} 82^{\circ} 38^{\prime} 12.28^{\prime \prime}\end{array}$ & 1570 & Nilka ilçesi yü zan \\
\hline 15 & 08.07 .2014 & $\begin{array}{l}\mathrm{N} 44^{\circ} 05^{\prime} 54.56^{\prime \prime} \\
\mathrm{E} 82^{\circ} 15^{\prime} 51.41^{\prime \prime}\end{array}$ & 1980 & Gulca ilçesi Meng ma la jia ba fang \\
\hline 16 & 10.07.2014 & $\begin{array}{l}\mathrm{N} 44^{\circ} 09^{\prime} 37.52^{\prime \prime} \\
\mathrm{E} 82^{\circ} 45^{\prime} 11.46^{\prime \prime}\end{array}$ & 2000 & Gulca ilçesi A wu zan gou \\
\hline 17 & $\begin{array}{l}12.07 .2014 \\
13.07 .2014\end{array}$ & $\begin{array}{l}\mathrm{N} 44^{\circ} 23^{\prime} 61.77^{\prime \prime} \\
\mathrm{E} 81^{\circ} 04^{\prime} 01.17^{\prime \prime}\end{array}$ & $1680-2400-3020$ & Korgas ilçesi guo zi gou \\
\hline 18 & 12.07 .2014 & $\begin{array}{l}\mathrm{N} 44^{\circ} 26^{\prime} 24.72^{\prime \prime} \\
\mathrm{E} 80^{\circ} 47^{\prime} 13.42^{\prime \prime}\end{array}$ & 1160 & Korgas ilçesi da xi gou \\
\hline 19 & 08.06 .2015 & $\begin{array}{l}\mathrm{N} 42^{\circ} 31^{\prime} 51.90^{\prime \prime} \\
\mathrm{E} 80^{\circ} 44^{\prime} 46.12^{\prime \prime}\end{array}$ & 2310 & Moğolküre ilçesi xia ta gu cheng \\
\hline 20 & 10.06 .2015 & $\begin{array}{l}\mathrm{N} 43^{\circ} 20^{\prime} 59.99^{\prime \prime} \\
\mathrm{E} 82^{\circ} 1621.13^{\prime \prime}\end{array}$ & 1252 & Dokuztara ilçesi Ye he tao gou \\
\hline 21 & $\begin{array}{l}11.06 .2015 \\
12.06 .2015 \\
\end{array}$ & $\begin{array}{l}\mathrm{N} 43^{\circ} 05^{\prime} 02.03^{\prime \prime} \\
\mathrm{E} 82^{\circ} 41^{\prime} 04.95^{\prime \prime}\end{array}$ & $1477-1632-1795$ & Dokuztara ilçesi çia şi \\
\hline
\end{tabular}

Bitki örneklerinin teşhis edilmesinde çeşitli flora ve makaleler kullanılmıştır (Gao Chien ve ark., 2010; Wu Peng Cheng ve ark., 2012; Gao Chien ve Wu Yu-Huan, 2008; Gao Chien, 2001; Keçeli, 2004; Şimşek, 2012; Sulayman, 2012a,b; Whittemore, ve ark., 1998; Grolle ve Long, 2000). Araştırma sonucu tespit edilen ciğerotu türlerinin otör isimlerinin yazılmasında başlıca yukarıda bahsedilen eserlerden http://www.theplantlist.org internet sitesinden faydalanılmıştır (URL2). Teşhis sırasında örneklerin ayrıt edici ve karakteristik özellikleri Nikon ECLIPSE E100 marka 1şık mikrokobu ve OLYMPUS SZ2ILST markalı diseksiyon mikroskobu kullanılarak gözlemlenmiştir (Şekil 2-20, Şekil 21-41). Türler alfabetik sırada verilmiştir. 

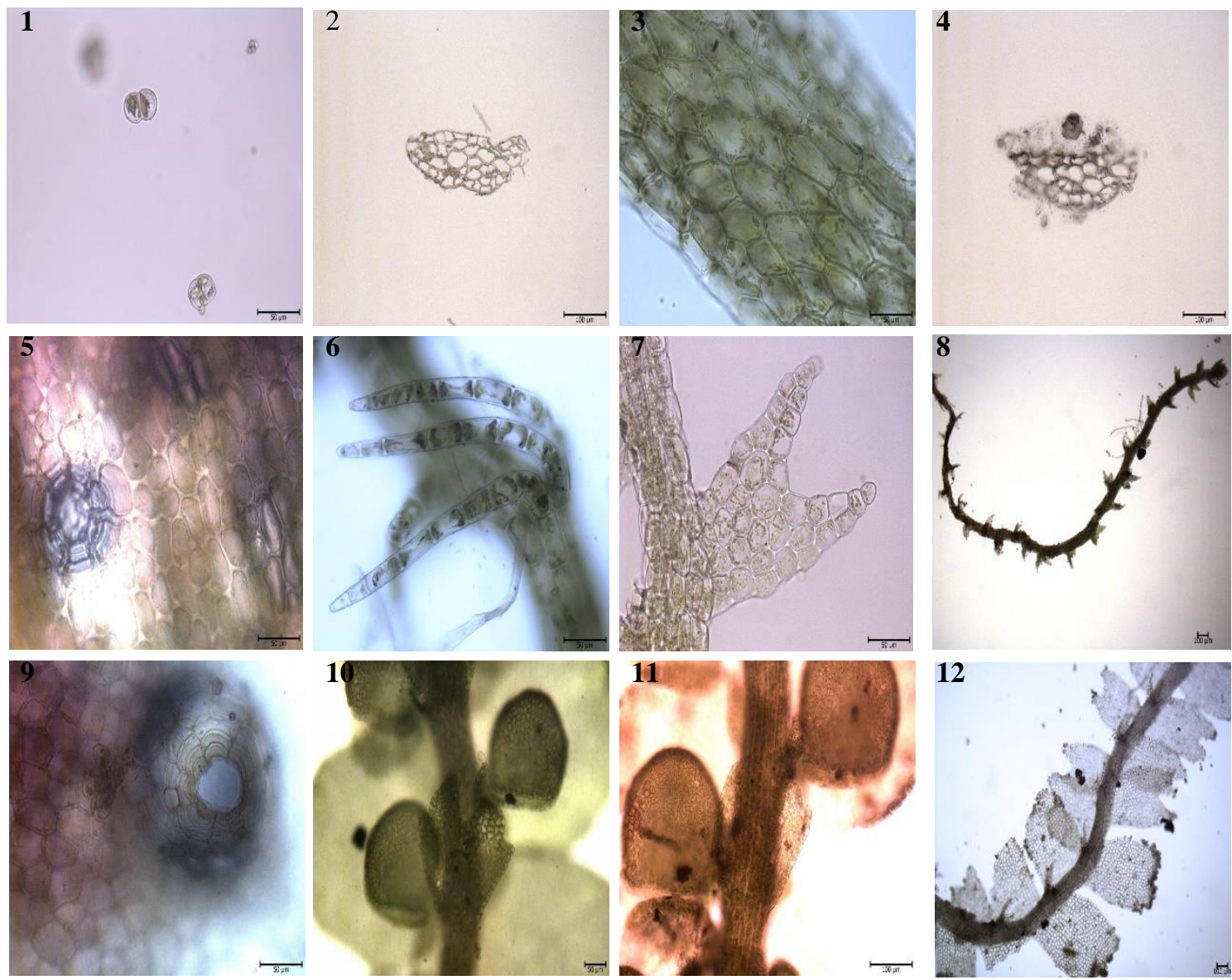

13
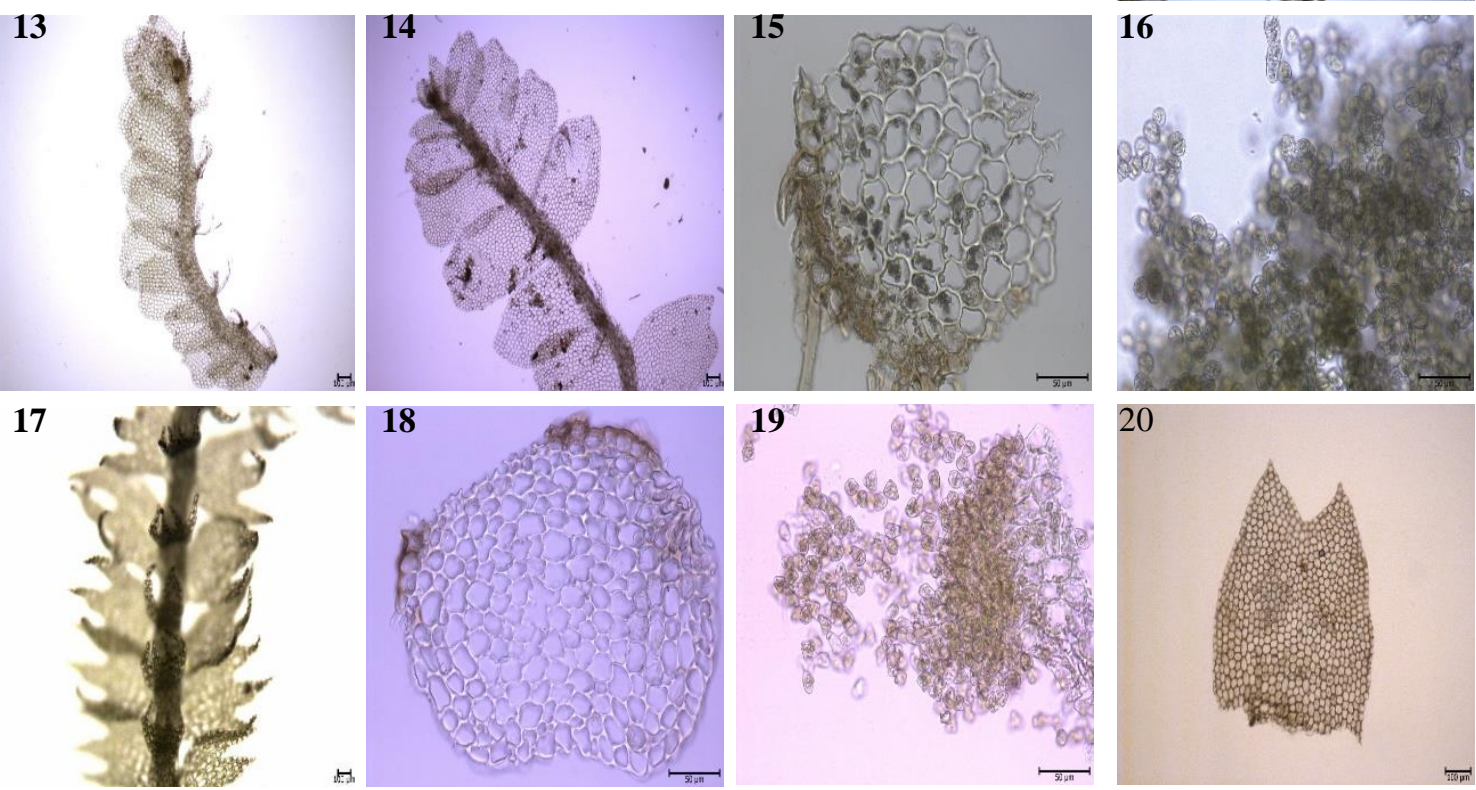

20

Şekil 2-20. 1. Riccardia chinensis, gemmalar, 2. R. latifrons, tallusun enine kesit, 3. R. miyakeana, tallusun üst yüzeyindeki epidermal hücreler, 4. R. palmata, tallusun enine kesiti, 5. Plagiochasma rupestre, tallusun üst yüzeyindeki epidermal hücreler ve trigonlar, 6. Blepharostoma trichophyllum, yaprak hücreleri, 7. Cephalozia lacinulata, yaprak şekli ve hücre görünüşü, 8. Cephaloziella hampeaana, bitkinin genel görünüşü, 9. Conocephalum conicum, tek bir hava porunun büyütülmüş şekli, 10. Frullania dilatata, alt yaprak ve miğfer şeklindeki ventral yaprak görünümü, 11. F. parvistipula, yaprak ve miğfer şeklindeki 
ventral yaprak görünümü, 12. Lohocolea minor, bitkinin ventral görünümü, 13. C. polyanthos, bitkinin ventral görünümü, 14. C. profundus, bitkinin ventral görünümü, 15. Syzygiella autumnalis, gövde enine kesit, 16. Jungermannia leiantha, gemmalar, 17. Lepidozia reptans, bitkinin ventral görünümü ve alt yaprak, 18. Barbilophozia barbata, gövdenin enine kesit, 19. Lophozia ascendens, gemmalar, 20. L. collaris, yaprak.

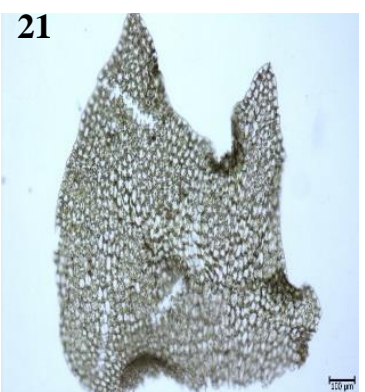

25

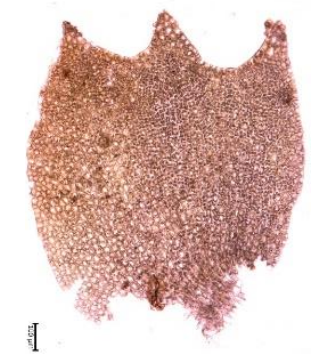

29
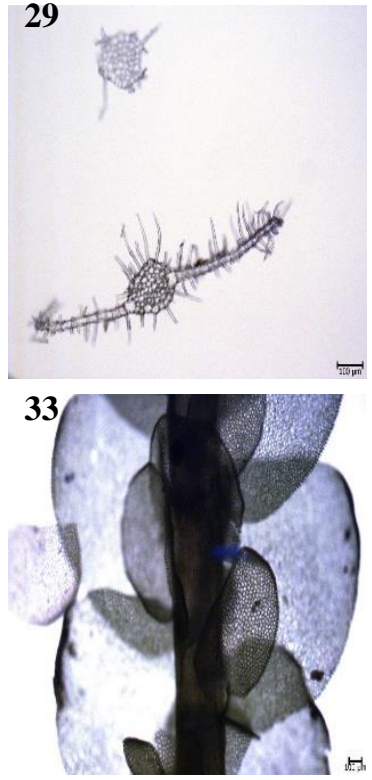

37

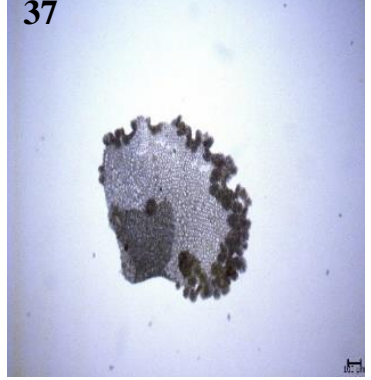

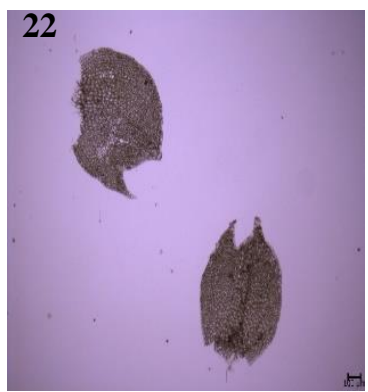
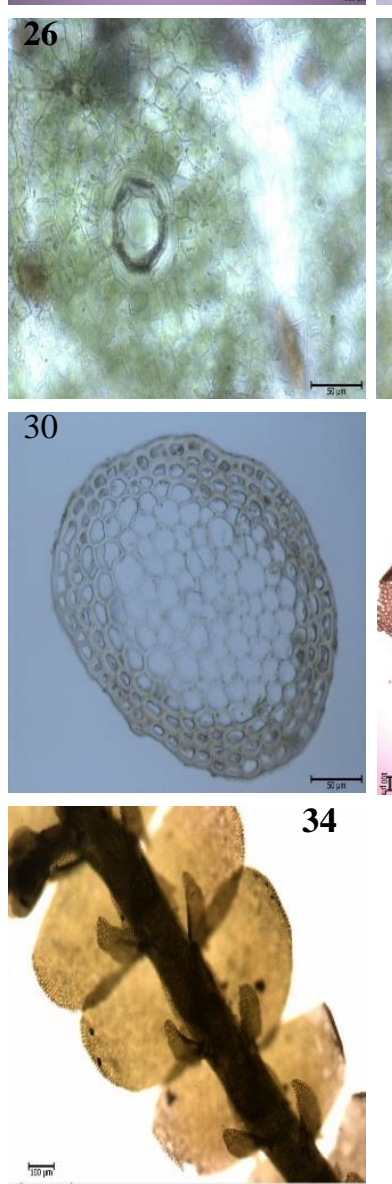

38

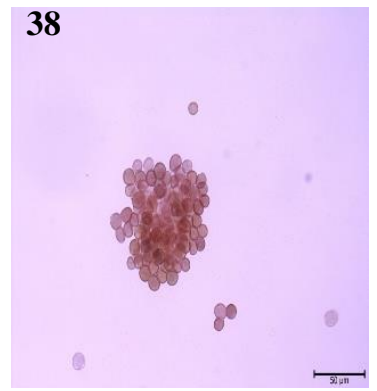

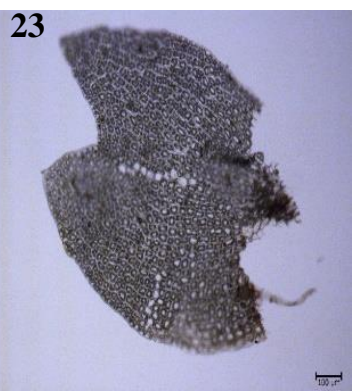
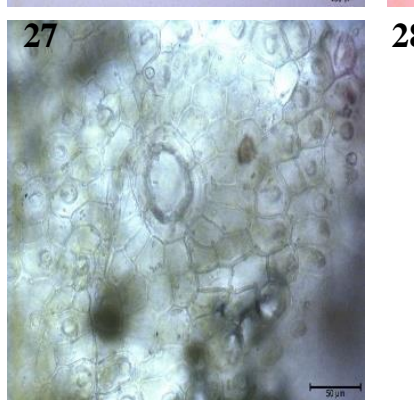

31

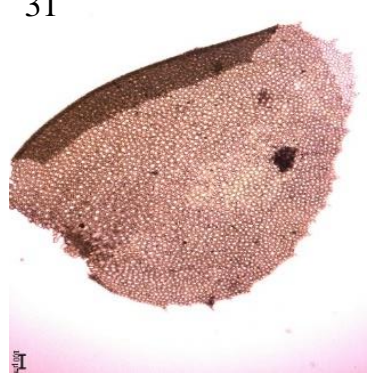

35

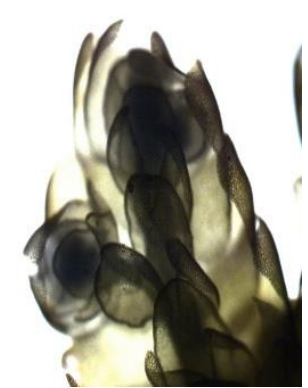

39

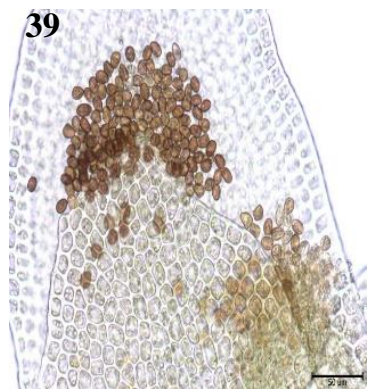

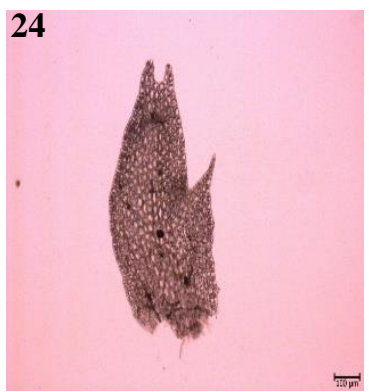

28
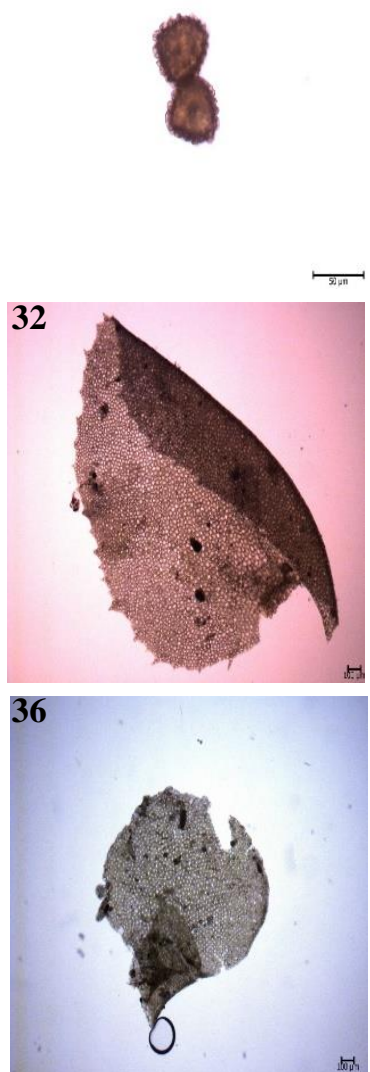

40

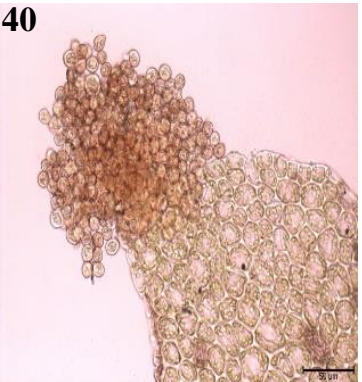




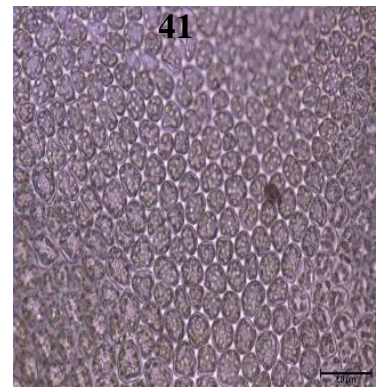

Şekil 21-41. 21. Lophozia excisa, yaprak, 22. L. sudetica, yaprak, 23. L. ventricosa, yaprak, 24. Tritomaria exsectiformis, yaprak, 25. T. quinquedentata, yaprak 26. Marchantia paleacea, hava poru, 27. M. polymorpha, d. hava poru, 28. Preissia quadrata, sporlar 29. Apometzgeria pubescens, tallusun enine kesit, 30. Plagiochila duthiana, gövde enine kesit, 31. P. porelloides, yaprak, 32. P. vexans, yaprak, 33. Porella obtusata, sürgünün ventral görünümü, 34. P. pinnata, sürgünün ventral kısm1, 35. P. platyphylla, sürgünün ventral k1sm1, 36. Radula complanata, yaprak, 37. R. lindenbergiana, yaprak, 38. Scapania apiculata, gemmalar, 39. S. carinthiaca, gemmalar, 40. S. glaucocephala, gemmalar, 41. Scapania parvifolia, yaprak hücreleri.

\section{Bulgular}

Bu çalışmada; 2012- 2015 yılları arasında Sincan Uygur Özerk Bölgesi, Sincan Tanrı Dağı'nın batısından toplanan 323 ciğerotu örneği teşhis edilmiş ve toplam 17 familyaya ait 21 cinse ait 41 tür tespit edilmiştir (Tablo 2).

Tablo 2: Familya, cins, tür, örnek sayıları ve istasyon no.

\begin{tabular}{|c|c|c|c|c|}
\hline Familya & Cins & Tür & $\begin{array}{l}\text { Örnek } \\
\text { Sayısı }\end{array}$ & $\begin{array}{c}\text { İstasyon } \\
\text { No } \\
\end{array}$ \\
\hline \multirow{4}{*}{ Aneuraceae } & \multirow{4}{*}{ Riccardia } & Riccardia chinensis C. Gao & 1 & 8 \\
\hline & & $\begin{array}{l}\text { Riccardia latifrons (Lindb.) } \\
\text { Lindb. }\end{array}$ & 1 & 3 \\
\hline & & $\begin{array}{l}\text { Riccardia latifrons var. } \\
\text { miyakeana (Schiff n.) Furuki }\end{array}$ & 1 & 3 \\
\hline & & $\begin{array}{l}\text { Riccardia palmata (Hedw.) } \\
\text { Carruth. }\end{array}$ & 2 & 6,8 \\
\hline Aytoniaceae & Plagiochasma & $\begin{array}{l}\text { Plagiochasma rupestre (G. Forst.) } \\
\text { Stephani }\end{array}$ & 4 & $1,2,3$ \\
\hline $\begin{array}{l}\text { Belpharastormataceae } \\
\text { (Pseudolepicoleaceae) }\end{array}$ & Blepharostoma & $\begin{array}{l}\text { Blepharostoma trichophyllum (L.) } \\
\text { Dumort. }\end{array}$ & 2 & 8,17 \\
\hline Cephaloziaceae & Cephalozia & $\begin{array}{l}\text { Cephalozia lacinulata (J.B. Jack } \\
\text { ex Gottsche \& Rabenh.) Spruce }\end{array}$ & 1 & 3 \\
\hline Cephaloziellaceae & Cephaloziella & $\begin{array}{l}\text { Cephaloziella hampeana (Nees) } \\
\text { Schiffner ex Loeske }\end{array}$ & 1 & 2 \\
\hline Conocephalaceae & Conocephalum & $\begin{array}{l}\text { Conocephalum conicum (L.) } \\
\text { Underw. }\end{array}$ & 13 & $\begin{array}{l}1,2,5,8 \\
16,21,20\end{array}$ \\
\hline \multirow{2}{*}{ Frullaniaceae } & \multirow{2}{*}{ Frullania } & Frullania dilatata (L.) Dumort. & 4 & $3,7,8$ \\
\hline & & Frullania parvistipula Stephani & 1 & 10 \\
\hline \multirow{3}{*}{ Geocalycaceae } & \multirow[t]{2}{*}{ Lophocolea } & Lophocolea minor Nees & 70 & $\begin{array}{c}1,2,5,6, \\
8,17,19, \\
21\end{array}$ \\
\hline & & $\begin{array}{l}\text { Lophocolea heterophylla } \\
\text { (Schrad.) Dumort. }\end{array}$ & 2 & 12,21 \\
\hline & Chiloscyphus & $\begin{array}{l}\text { Chiloscyphus polyanthos (L.) } \\
\text { Corda }\end{array}$ & 3 & $8,12,21$ \\
\hline
\end{tabular}




\begin{tabular}{|c|c|c|c|c|}
\hline Jungermanniaceae & Syzygiella & $\begin{array}{l}\text { Syzygiella autumnalis (DC.) } \\
\text { K.Feldberg, Váňa, Hentschel et } \\
\text { Heinrichs }\end{array}$ & 1 & 6 \\
\hline & Jungermannia & Jungermmannia leiantha Grolle & 1 & 9 \\
\hline Lepidoziaceae & Lepidozia & Lepidozia reptans (L.) Dumort. & 26 & $\begin{array}{l}2,3,5,6 \\
8,9,10 \\
14,19,21\end{array}$ \\
\hline \multirow{8}{*}{ Lophoziaceae } & Barbilophozia & $\begin{array}{l}\text { Barbilophozia barbata (Schreb.) } \\
\text { Loeske }\end{array}$ & 2 & 3 \\
\hline & \multirow{2}{*}{ Lophozia } & $\begin{array}{l}\text { Lophozia ascendens (Warnst.) } \\
\text { R.M. Schust. }\end{array}$ & 1 & 8 \\
\hline & & $\begin{array}{l}\text { Lophozia ventricosa (Dicks.) } \\
\text { Dumort. }\end{array}$ & 1 & 2 \\
\hline & Lophoziopsis & $\begin{array}{l}\text { Lophoziopsis excisa (Dicks.) } \\
\text { Konstant. et Vilnet }\end{array}$ & 1 & 13 \\
\hline & Mesoptychia & $\begin{array}{l}\text { Mesoptychia collaris (Nees) } \\
\text { L.Söderstr. et Váňa }\end{array}$ & 2 & 6,17 \\
\hline & Barbilophozia & $\begin{array}{l}\text { Barbilophozia sudetica (Nees ex } \\
\text { Huebener) L.Söderstr. }\end{array}$ & 9 & $6,8,13,17$ \\
\hline & Tritomaria & $\begin{array}{l}\text { Tritomaria exsectiformis (Breidl.) } \\
\text { Schiffner ex Loeske }\end{array}$ & 2 & 2,19 \\
\hline & Trilophozia & $\begin{array}{l}\text { Trilophozia quinquedentata } \\
\text { (Huds.) Bakalin, }\end{array}$ & 3 & 3,17 \\
\hline \multirow{3}{*}{ Marchantiaceae } & \multirow[b]{2}{*}{ Marchantia } & Marchantia paleacea Bertol. & 2 & 2,8 \\
\hline & & Marchantia polymorpha L. & 25 & $\begin{array}{c}1,3,9,10 \\
17,21\end{array}$ \\
\hline & Preissia & Preissia quadrata (Scop.) Nees & 10 & $1,3,5,7,8$ \\
\hline Metzgeriaceae & Apometzgeria & $\begin{array}{l}\text { Metzgeria pubescens (Schrank) } \\
\text { Raddi }\end{array}$ & 8 & $\begin{array}{c}6,10,11 \\
13\end{array}$ \\
\hline \multirow{3}{*}{ Plagiochilaceae } & \multirow{3}{*}{ Plagiochila } & Plagiochila duthiana Stephani & 2 & 6,9 \\
\hline & & $\begin{array}{l}\text { Plagiochila porelloides (Torr. ex } \\
\text { Nees) Lindenb. }\end{array}$ & 20 & $\begin{array}{c}2,6,8,11 \\
21\end{array}$ \\
\hline & & $\begin{array}{l}\text { Plagiochila vexans Schiffner ex } \\
\text { Stephani }\end{array}$ & 5 & $2,5,10$ \\
\hline \multirow{3}{*}{ Porellaceae } & \multirow{3}{*}{ Porella } & Porella obtusata (Taylor) Trevis. & 13 & $\begin{array}{c}2,6,7,16 \\
18\end{array}$ \\
\hline & & Porella pinnata $\mathrm{L}$. & 3 & 10 \\
\hline & & Porella platyphylla (L.) Pfeiff. & 6 & $\begin{array}{c}1,2,3,9 \\
10,16\end{array}$ \\
\hline \multirow{2}{*}{ Radulaceae } & \multirow{2}{*}{ Radula } & Radula complanata (L.) Dumort. & 52 & $\begin{array}{c}1,3,8,14 \\
16,18\end{array}$ \\
\hline & & $\begin{array}{l}\text { Radula lindenbergiana Gottsche } \\
\text { ex Hartm. }\end{array}$ & 10 & $\begin{array}{c}2,3,6,8 \\
10,12\end{array}$ \\
\hline \multirow{4}{*}{ Scapaniaceae } & \multirow{4}{*}{ Scapania } & Scapania apiculata Spruce & 1 & 12 \\
\hline & & $\begin{array}{l}\text { Scapania carinthiaca J.B. Jack ex } \\
\text { Lindb. }\end{array}$ & 1 & 13 \\
\hline & & $\begin{array}{l}\text { Scapania glaucocephala (Taylor) } \\
\text { Austin }\end{array}$ & 5 & $8,9,12$ \\
\hline & & Scapania parvifolia Warnst. & 5 & $\begin{array}{c}2,3,7,8 \\
12,17\end{array}$ \\
\hline
\end{tabular}




\section{Tartışma ve Sonuç}

Araştırma alanında bulunan türlerin familyalara göre dağılımı da Tablo 3'de verilmiştir.

Familyaların içerdiği tür sayılarına baktığımızda en çok türün 8 tür ile Lophoziaceae familyasında olduğu görülür. $\mathrm{Bu}$ familyaya ait türlerin tüm türlere oranı \%19,50'dir. İkinci olarak ise en çok tür sayısı içeren familya 4 türle Aneuraceae, Scapaniaceae familyalarıdır ve tüm türlere oranı ise $\% 9,75^{\prime} \mathrm{d}$ ir.

Bu iki familyadan sonra sırasıyla Geocalycaceae, Marchantiaceae, Plagiochilaceae, Porellaceae familyaları 3'er tür ile \%7,32, Frullaniaceae, Jungermanniaceae, Radulaceae familyaları 3'er tür ile $\% 4,88, \quad$ Aytoniaceae, Belpharastormataceae, Cephaloziaceae, Cephaloziellaceae, Conocephalaceae, Lepidoziaceae, Metzgeriaceae familyaları birer tür ile \%2,44 oranlarında olduğu görülmektedir. Familyalara ait tür sayıları ve familyalara ait tür sayılarının toplam tür sayısına oranları Tablo 3 'te gösterilmiştir.

Araştırma alanından toplanan ciğerotlarının toplandığg substrat tipilerine göre değerlendirmesi yapılmış ve sonuçlar; Şekil 5'de belirtilmiştir. Teşhis edilen türlerin substrat tipleri ve bu substrat tiplerine ait olan örnek sayıları belirlenmiştir. Her bir substrat tipine ait olan örnek sayılarının toplam örnek sayısına oranları hesaplanmıştır. Buna göre toplanan tüm örnekleden 142 tanesi Ölü Ağaç Kütükleri üzerinden(Ö) toplanmış ve toplanan tüm örneklerin \%43,61'ini oluşturmaktadır ve bu oranla en çok örnek toplanan substrat tipi olarak belirlenmiştir. Diğer örneklerin substrat sırası ise 95 örnek $(\% 29,60)$ Kaya Üzerinden $(\mathrm{K}), 51$ örnek(\%15,89) Toprak Üzerinden(T), 23 örnek ( \%7,16) Ağaç Üzerinden (A), 11 örnek $(\% 3,43)$ Islak Toprak Üzerinden (IT) ve 1 örnek $(\% 0,31)$
Islak Kaya Üzerinden (IK) toplanmış olarak en son sirada gelmektedir.

Tablo 3. Çalışma kapsamında toplanmış olan türlerin familyalara göre dağılımları

\begin{tabular}{|l|c|c|}
\hline \multicolumn{1}{|c|}{ Familya } & $\begin{array}{c}\text { Tür } \\
\text { Tär } \\
\text { Sayısııı }\end{array}$ & $\begin{array}{c}\text { Toplam Tür } \\
\text { Sayısına } \\
\text { Oranı (\%) }\end{array}$ \\
\hline Lophoziaceae & 8 & 19,50 \\
\hline Aneuraceae & 4 & 9,75 \\
\hline Scapaniaceae & 4 & 9,75 \\
\hline Geocalycaceae & 3 & 7,32 \\
\hline Marchantiaceae & 3 & 7,32 \\
\hline Plagiochilaceae & 3 & 7,32 \\
\hline Porellaceae & 3 & 7,32 \\
\hline Frullaniaceae & 2 & 4,88 \\
\hline Jungermanniaceae & 2 & 4,88 \\
\hline Radulaceae & 2 & 4,88 \\
\hline Aytoniaceae & 1 & 2,44 \\
\hline Belpharastormataceae & 1 & 2,44 \\
\hline Cephaloziaceae & 1 & 2,44 \\
\hline Cephaloziellaceae & 1 & 2,44 \\
\hline Conocephalaceae & 1 & 2,44 \\
\hline Lepidoziaceae & 1 & 2,44 \\
\hline Metzgeriaceae & 1 & 2,44 \\
\hline TOPLAM: & $\mathbf{4 1}$ & $\mathbf{1 0 0}$ \\
\hline
\end{tabular}




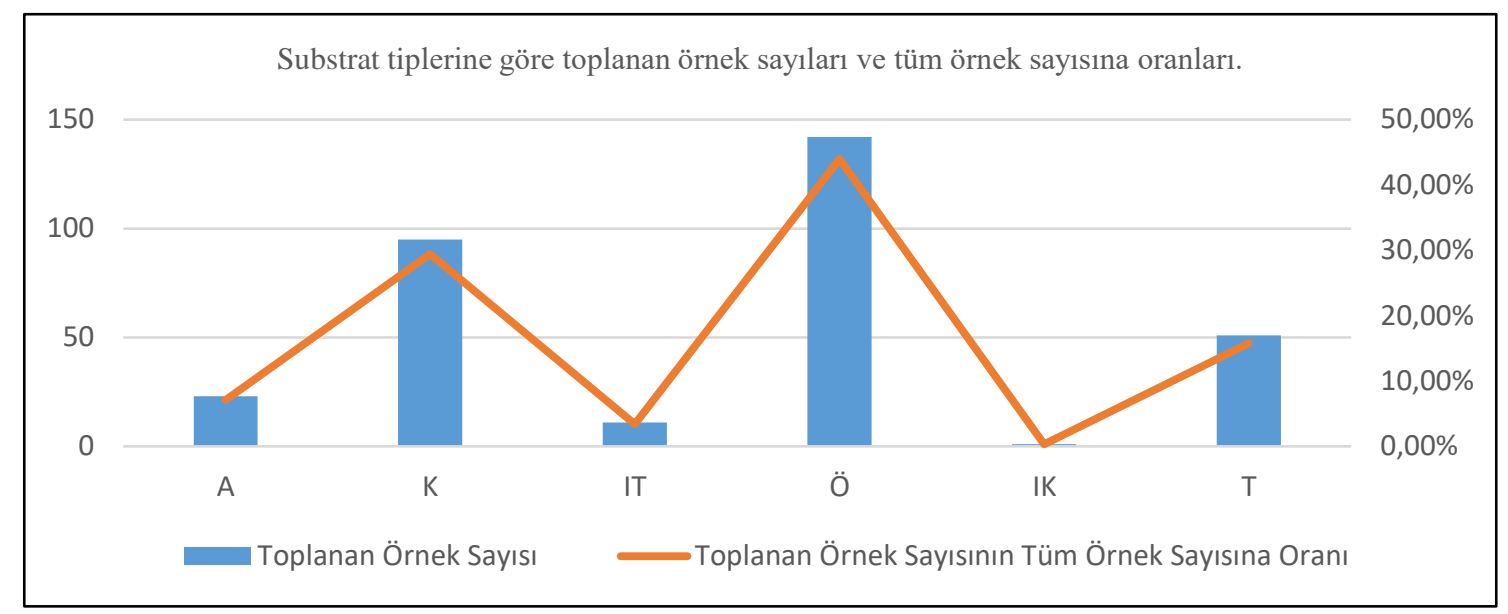

Şekil 5: Substrat tiplerine göre toplanan örnek sayıları ve tüm örnek sayısına oranlarının sütun grafiği.

Araştırma alanı ve Tablo 4 de elde edilen sonuçlar göre araştırma bölgesinde en çok türün 8 tür ile Lophoziaceae familyasında olduğu görülür. $\mathrm{Bu}$ familyaya ait türlerin tüm türlere oranı \%19,50'dir. İkinci olarak ise en çok tür sayısı içeren familya 4 türle Aneuraceae ve Scapaniaceae familyalarıdır ve bu familyaların tüm türlere oranı ise $\% 9,75$ ' dır.

Araştırma alanına yakın bölgelerde yapılmış çalışmalarla karşılaştırıldığında da karşımıza benzer neticeler çıkar. Abdugeni, G (2015) yaptığ çalışmada Doğu Tanrı Dağları ciğerotları florasını çalışmış ve 72 tür teşhis etmiştir. Bu çalışma ile Abdugeni, G (2015) çalışması karşılaştırıldığında (Tablo 4) tür sayısı fazla olmasına rağmen benzer oranların elde edilmiş olduğu görülür. $\mathrm{Bu}$ bulgu, söz konusu iki bölge fitocoğrafik bakımdan farklılık göstermediğinden ilgi çekicidir. $\mathrm{Bu}$ çalışmada en fazla türün bulunduğu familyanın, 14 tür ile Lophoziaceae olduğu, bu familyaya ait türlerin tüm türlere oranı \%19,44 ile en çok tür barındıran familya olduğu verilmiştir. İkinci olarak ise en çok tür sayısı içeren familya 7 türle Jungermanniaceae familyası ve tüm türlere oranı ise $\% 9,72$ olarak verilmiştir

Tablo 4. Tür sayılarının familyalara göre dağılımının araştırma alanına yakın bölgelerde yapılmış çalışmalar ile karşılaştırılması

\begin{tabular}{|c|c|c|c|c|c|c|c|c|c|c|}
\hline & \multirow{2}{*}{\multicolumn{2}{|c|}{$\begin{array}{c}\text { Batı Tanrı } \\
\text { Dağları }\end{array}$}} & \multicolumn{2}{|c|}{$\begin{array}{c}\text { Doğu Tanrı } \\
\text { Dağları } \\
\text { (Abdugeni, } \\
\text { 2015) }\end{array}$} & \multicolumn{2}{|c|}{$\begin{array}{c}\text { Kanas Doğa } \\
\text { koruma } \\
\text { alanı } \\
\text { (Sulayman, } \\
\text { 1999b) }\end{array}$} & \multicolumn{2}{|c|}{$\begin{array}{c}\text { Boğda Dağı } \\
(\text { Zhao, 1993) }\end{array}$} & \multirow{2}{*}{\multicolumn{2}{|c|}{$\begin{array}{c}\text { Sangong } \\
\text { Nehir } \\
\text { bölgesi } \\
\text { (Zhang ve } \\
\text { ark, 2002a) }\end{array}$}} \\
\hline Toplam Tak. S. & & & & & & & & & & \\
\hline Familya & $\begin{array}{c}\text { Tak. } \\
\text { S. }\end{array}$ & $\%$ & $\begin{array}{c}\text { Tak. } \\
\text { S. }\end{array}$ & $\%$ & $\begin{array}{c}\text { Tak. } \\
\text { S. }\end{array}$ & $\%$ & $\begin{array}{c}\text { Tak. } \\
\text { S. }\end{array}$ & $\%$ & $\begin{array}{c}\text { Tak. } \\
\text { S. }\end{array}$ & $\%$ \\
\hline Lophoziaceae & 8 & 19,50 & 14 & 19,44 & 4 & 19,04 & 4 & 17,39 & 4 & 16,67 \\
\hline Aneuraceae & 4 & 9,75 & 5 & 6,94 & 3 & 14,29 & - & - & - & - \\
\hline Scapaniaceae & 4 & 9,75 & 6 & 8,33 & - & - & 3 & 13,04 & 3 & 12,50 \\
\hline Geocalycaceae & 3 & 7,32 & 2 & 2,78 & 2 & 9,52 & 1 & 4,35 & 1 & 4,17 \\
\hline Marchantiaceae & 3 & 7,32 & 3 & 4,71 & 1 & 4,76 & 1 & 4,35 & 1 & 4,17 \\
\hline Plagiochilaceae & 3 & 7,32 & 6 & 8,33 & 1 & 4,76 & 2 & 8,69 & 2 & 8,33 \\
\hline Porellaceae & 3 & 7,32 & 6 & 8,33 & 1 & 4,76 & 3 & 13,04 & 3 & 12,50 \\
\hline Frullaniaceae & 2 & 4,88 & 1 & 1,39 & - & - & - & - & - & - \\
\hline Jungermanniaceae & 2 & 4,88 & 7 & 9,72 & - & - & 2 & 8,69 & 2 & 8,33 \\
\hline Radulaceae & 2 & 4,88 & 3 & 4,17 & 1 & 4,76 & 1 & 4,35 & 1 & 4,17 \\
\hline
\end{tabular}


Sulayman (1999b) Kanas Doğa Koruma Alanı Bryofit florasını çalışmış ve bölgeden 21 ciğerotu bildirmiştir. Yaptığı bu çalışmada \%19,04 oran ile Lophoziaceae (4 tür) en çok tür barındıran familya konumundadır. İkinci konumda ise 3 tür ve $\% 14,29$ oran ile Aneuraceae familyası olduğu bildirilmiştir.

Zhao (1993a) Boğda Dağı Bryofit florasını çalışmış ve bölgeden 23 ciğerotu türü bildirmiştir. Bu çalışmada 4 tür ve $\% 17,39$ oran ile en çok tür içeren Lophoziaceae ilk familya konumundadır. İkinci konumda ise 3 tür ve $\% 13,04$ oran ile Scapaniaceae olduğu bildirilmiştir.

Zhang ve ark., (2002a) tarafindan Sangong Nehir bölgesinde araştırma yapılmış ve 24 tür ciğerotu bildirilmiştir. Bu çalışmada $\% 16,67$ oran ve 4 tür ile ilk sirada yine Lophoziaceae familyas gelmektedir. İkinci surada ise 3 tür ve $\% 12,50$ oran ile Scapaniaceae olduğu bildirilmiştir.

$\mathrm{Bu}$ çalışma sonucunda elde edilen verilerin gelecekte yapılacak olan gerek floristik gerekse ekolojik çalışmalara yardımcı olacağı ve ilerde yazılacak olan Sincan Uygur Özerk Bölgesi Hepatophyta Florası'na katkı sağlayacağı düşünülmektedir.

\section{Teșekkür}

Bu çalışma, ilk yazarın yüksek lisans tezinden üretilmiş olup, çalışmamda konu, kaynak ve yöntem açısından yol gösteren Prof. Dr. Mamtimin SÜLEYMAN'a (Xinjiang Üniversitesi, Çin), bazı örneklerin teşhislerinin doğrulanmasında yardımları olan Prof. Dr. Mesut KIRMACI, Prof. Dr. Hatice ÖZENOĞLU 'na yürekten teşekkür ederim. Ayrıca Uzman Yusuf Can GERÇEK ve Dr. Almila ÇİFTÇİ'YE yürekten teşekkür ederim.

\section{Kaynaklar}

Abdugeni G. 2015. Flora and Taxanomy of Liverworts in Eastern Tianshan, Xinjiang. Thesis. Xinjiang University College of Life Science and Technology. Xinjiang.

Gao C. 2001. Flora Bryophytorum Sinicorum Vol.9. Science Press. Beijing.

Gao C. Wu Y.H. 2008. Flora Bryophytorum Sinicorum Vol.10. Science Press. Beijing.

Gao C. Wu Y.H. 2010. Genera Hepaticopsida et Anthocerotopsida Sinicorum. Science Press. Beijing.

Gupur R. Abdurehim A. Ablimit M. Sulayman M. 2014. Recent advances in Xinjiang Bryological Research. Journal of Xinjiang University. 31:3, 335-340.
Grolle R. Long D.G. 2000. An annotated check list of the Hepaticae and Anthocerotae of Europae and Macaronesia. Journal of Bryology. 22,103-140.

Hu Ru Ji ve ark. 2004. Physical Geography of The Tianshan Mountains İn China. China Environmental Science Press. Beijing.

Keçeli T. 2004. Batı Karadeniz Bölgesi (BoluZonguldak-Bartın-Kastamonu) Ciğerotları (HEPATICAE) Florasi. Doktora Tezi. Ankara Üniversitesi Fen Bilimleri Enstitüsü Biyoloji Anabilim Dalı. Ankara.

Şimşek Ö. 2012. Bolu Dağları Ciğerotları (Hepaticae) Florası. Doktora Tezi. Ankara Üniversitesi Fen Bilimleri Enstitüsü Biyoloji Anabilim Dalı. Ankara.

Sulayman M. 2012a. The Eco-geographical Distribution Pattern of Bryophytes from Karakorum-West Aria in Xinjiang. Journal of Xinjiang University. 29:2,229-234.

Sulayman M. 2012b. New Checklist of Xinjiang Liverworts, Hornworts and Mosses. Journal of Xinjiang University. 29:3, 259267.

Sulayman M. Zhao J.C. \& Sop hia. 1999b. A Study of the Bryoflora of the Kanas Nature Reserve, Xinjiang. Arid Zone Res. 16:4, 19-24.

URL1. Wikiwand. 2017. Website: http://www.wikiwand.com/tr/\%C4\%B0li_ Kazak_\%C3 \%96zerk_\%C4\%B01i [Erişim: 15 Ekim 2017].

URL2. The Plant List 2010. Website: http://www.theplantlist.org/ [Erişim: 16 Temmuz 2017].

Whittemore A.T. Zhu R.L. Hu R.L Zhao J.C. 1998. A checklist of Liverworts of Xinjiang, China. The Bryologist. 101:3, 439-443.

Wu Peng Cheng ve ark. 2012. Higher Plants Of China Volume 1. Qingdao Publishing House. Qingdao.

Yang Chang You. 1993. Flora Xinjiangensis. Xinjiang Science \& Technology \& Hygiene Publishing House. Xinjiang.

Zhang Y.M. Cao T. Pan B.R. 2002a. Study on Bryophytes and Their Distribution Patterns in Sangong River Watershed, Xinjiang. Arid Zone Res. 19:1, 39-43.

Zhang Y.M. 2002. Study on bryophyte flora and ecology in Sangong River Watershed, Xinjiang. Ph.D. Thesis. Chinese Academy of Sciences.

Zhao J C. 1993a. Study on the bryophytes on Mt. Bogda, Xinjiang. Journ of Xinjiang Univ. 10:1, 73-92. 